\title{
The Prevalence and associated Factors for Liver Metastases, Development and Prognosis in newly diagnosed Epithelial Ovarian Cancer: A large Population-Based Study from the SEER Database
}

\author{
Iftikhar Hussain*, Jiaqin Xu*, Kui Deng, Noor-ul-Amin, Ce Wang, Yue Huang, Shuang Li, Kang Li ${ }^{\bowtie}$ \\ Department of Epidemiology and Biostatistics, School of Public Health, Harbin Medical University, Harbin 150086, People's Republic of China. \\ *These authors contributed equally to this work. \\ $\triangle$ Corresponding author: Department of Epidemiology and Biostatistics, Public Health School, Harbin Medical University, Harbin 150086, People's Republic of \\ China. E-mail address: liking@ems.hrbmu.edu.cn (Kang Li). \\ (c) The author(s). This is an open access article distributed under the terms of the Creative Commons Attribution License (https://creativecommons.org/licenses/by/4.0/). \\ See http://ivyspring.com/terms for full terms and conditions.
}

Received: 2019.09.24; Accepted: 2020.03.27; Published: 2020.06.08

\begin{abstract}
Background: Primary Epithelial Ovarian Cancer (EOC), a malignant gynecologic disease, is considered one of the leading causes of mortality in women. The development of Liver Metastases (LM) in women with primary ovarian cancer commonly results in a poorer prognosis. This retrospective population-based study aims to measure the prevalence, prognostic factors, and associated risk factors for epithelial ovarian cancer patients with liver metastases (EOCLM).

Materials and Methods: The current study cohort of patients based on the Surveillance, Epidemiology, and End Results (SEER) database identified with primary ovarian cancer between the years 2010 and 2016. A chi-square test was employed to compare Metastatic differences among demographic and clinical factors. Univariable and multivariable logistic regression analysis models were used to predict related prognostic factors for LM development. 7-year Kaplan-Meier curves were applied to compare the survival patterns of patients with and without LM. The Multivariable Cox regression model was used to estimate potential risk factors associated with LM related deaths.

Results: 33895 eligible primary EOC patients were identified. Among them 2635 (7.77\%) patients were initially diagnosed with de novo LM, and 31260 (92.23\%) without metastases disease to any site. Non-serous histology type; Malignant Brenner Carcinoma, NOS (OR 1.94; Cl: 1.39-2.71; P<0.001), T3/T1 stage (OR 5.65; Cl: 3.87-8.24; $P<0.001$ ), N1/N0 stage (OR 1.67; Cl: 1.43-4.95; $P<0.001$ ), grade; G3/G1 (OR 2.16; $\mathrm{Cl}: 1.29-3.59, P<0.001$ ), and cancer antigen-125; Elevated/Normal (OR 1.79; $\mathrm{Cl}$ : 1.19-2.69, $P<0.001$ ) were significantly associated with LM occurrence. The median survival of EOC patients with LM was 12.0 (95\% Cl: 11.0-14.0; $P<0.001)$ months. Multivariable cox regression showed being unmarried $(\mathrm{HR}$ 1.16; $\mathrm{Cl}$ : 1.04-1.30; $P=0.001)$, non-serous histology types, Mucinous (HR 2.38; $\mathrm{Cl}$ : 1.82-3.12; $P<0.001)$, Clear cell (HR 1.83; Cl: 1.32-2.55; $P<0.001)$, Malignant Brenner Carcinoma, NOS (HR 1.44; Cl:1.23-1.66; $P<0.001)$, Carcinosarcoma NOS, $(\mathrm{HR} 1.44 ; \mathrm{Cl}$ : 1.11-1.88; $P<0.001)$ and radiotherapy (HR 1.52; $\mathrm{Cl}$ : 1.12-2.06; $P<0.001)$, were positively related to death. Chemotherapy (HR 0.30; Cl: 1.12-2.06; $P<0.001)$ and surgery (HR 0.34; $\mathrm{Cl}: 0.29-0.39 ; P<0.001)$ were related with reduced rate of death.

Conclusion: The retrospective cohort study showed that women with primary EOC had some high-risk factors associated with LM. LM can intensely decrease the survival of EOC patients. The findings of our research provided estimates for LM occurrence prediction and potential prognostic factors of EOC with de novo LM development. These findings can be useful for follow-up strategies, guidelines for screening, and treatment of EOCLM.
\end{abstract}

Key words: Liver Metastases, Epithelial Ovarian Cancer, SEER database, Survival, Risk factors, Prognostic factors 


\section{Introduction}

Epithelial Ovarian Cancer considered among the fatal gynecologic malignancies of the female reproductive system, with expected 22,530 new cases and 13,980 deaths to occur in 2019 in the US [1,2]. Ovarian cancer accounts for $2.5 \%$ of all fatalities among women. Almost $60 \%$ of EOC patients diagnosed at a distant stage. The 5-year survival of EOC patients is still less than $47 \%$ [3]. The lifetime risk of ovarian cancer incidence is $1.39 \%$, and the lifetime risk of death is $1.04 \%$ [2]. The majority of EOC patients diagnosed with advanced stages (III and IV) due to no practical screening tests and symptoms are distinct. About 12-33\% of EOC patients will be diagnosed with FIGO stage IV, de novo Metastases disease at initial diagnosis [4]. Liver Metastases found in up to $50 \%$ of patients dying of EOC $[5,6]$. Recent studies noted liver Metastases reported as the most common cause of stage-IV disease in EOC patients $[4,7]$. Hepatic resection of patients with primary ovarian cancer with liver metastases could benefit in terms of survival [8]. Perihepatic Metastases in advanced EOC patients occurs through the peritoneal spread of tumor implants on the liver surface. Sometimes, perihepatic metastases can invade the liver parenchymal [9]. Several studies suggest surgery and platinum-based chemotherapy as the primary treatments associated with overall survival $[10,11]$.

However, there are no LM screening guidelines for EOC patients with de novo LM. Hussain SM et al. stated that MRI is commonly used and considered optimal diagnostic modality for the assessment of alleged hepatic metastases [12]. Other diagnostic modalities that often used are CT and PET-CT. Still, it could not identify the metastatic tumors less than 1.0 $\mathrm{cm}$. also MRI, was not recommended for ovarian cancer patients' routine assessment in current guidelines for EOC patients screening $[13,14]$.

Clinico-demographic characteristics of EOCLM patients, in the early estimation of the prognostic factors associated with LM can help the physicians to develop treatment strategies. Identification of potential risk factors and the importance of different treatment plans on EOCLM need to be assessed to provide alternative care and guidelines for the treatment of EOCLM patients based on a large population. Nevertheless, some studies with a minimal number of ovarian cancer patients with liver metastases were published before.

The Surveillance, Epidemiology, and End Results (SEER) is a publicly available cancer database that covers $30 \%$ of the United States population. The SEER system routinely records data on patient demographics, tumor characteristics, tumor morphology and histology, general treatment, stage at diagnosis, survival time, and follow-up. The current study aimed to identify the risk factors associated with de novo LM in EOC and to inspect the prevalence and survival trends of EOCLM patients. The study covers patients between the years 2010 and 2016 since the details of Liver Metastases (LM) were not available before 2010 .

\section{Materials and Methods}

\section{Study Population}

Primary EOC patients diagnosed between the years 2010 and 2016 were identified using the National Cancer Institute's Surveillance, Epidemiology, and End Results (SEER) database [34]. Patients are meeting the following criteria: The site-recode ICD-O-3(International Classification of Disease for Oncology-3)/WHO 2008[15], primary site C56.9 restricted as "Ovary." Patients diagnosed between 2010 and 2016, aged $\geq 18$ years old; diagnosed with histologically confirmed invasive epithelial tumors and demographics (age, marital status, and race), clinicopathological parameters (stage, tumor grade, laterality, treatment details, survival and cause of death) included in the study cohort.

Patients diagnosed by death certificate or autopsy only, patients with unknown information of follow-up or LM and patients without LM but lung, Bone, and Brain metastases were excluded from the study cohort. The detailed selection procedure is summarized in Figure 1. All data were extracted using SEER.Stat 8.3 .5 software.

\section{Statistical Analysis}

Demographic variables including age, race, marital status, and insurance were classified as followed: Age ( $\leq 40,41-64$ and $\geq 65$ years), race (white, black and others), marital status (married and unmarried), and insurance status (insured, uninsured). Clinical variables, tumor location (one side, paired side and bilateral), cancer antigen-125 (normal and elevated), Tumor grade (I, II, III and IV), Tumor stage (T1, T2, and T3); regional lymph node (N0 and N1) defined according to the American Joint Committee on Cancer seventh edition (AJCC) [16]. Histology was characterized as serous, endometrioid, mucinous, clear cell, Malignant Brenner Carcinoma NOS, Carcinosarcoma, and others $[15,17]$.

Categorical variables are reported as counts (percentages). The chi-square test was used to analyze the differences between the subgroups. A $P$-value of less than 0.05 was considered statically significant. Univariable logistic regression was employed to predict potential risk factors for EOC patients with de novo LM. The factors statistically significant at the 
significance level of $<0.05$ were adjusted for further analysis using multivariable logistic regression.

The primary endpoint of the study cohort was to determine the overall survival of EOC patients with LM. A patient's survival was defined as the date of diagnosis until death from any cause. Kaplan-Meier curve method was employed to estimate overall survival, and the log-rank test was applied to evaluate the survival differences. Univariable Cox analysis was performed to investigate the prognostic factors for LM. Factors statistically significant at $(p<0.05)$, in Univariable Cox Regression analysis, were then further evaluated using the Multivariable Cox model to classify the prognostic factors associated with overall survival among EOC patients with LM. All statistical analyses are performed using $\mathrm{R}$ version R-3.6.1 and Kaplan Meier curves were drawn using the R package ggplot2 [18].

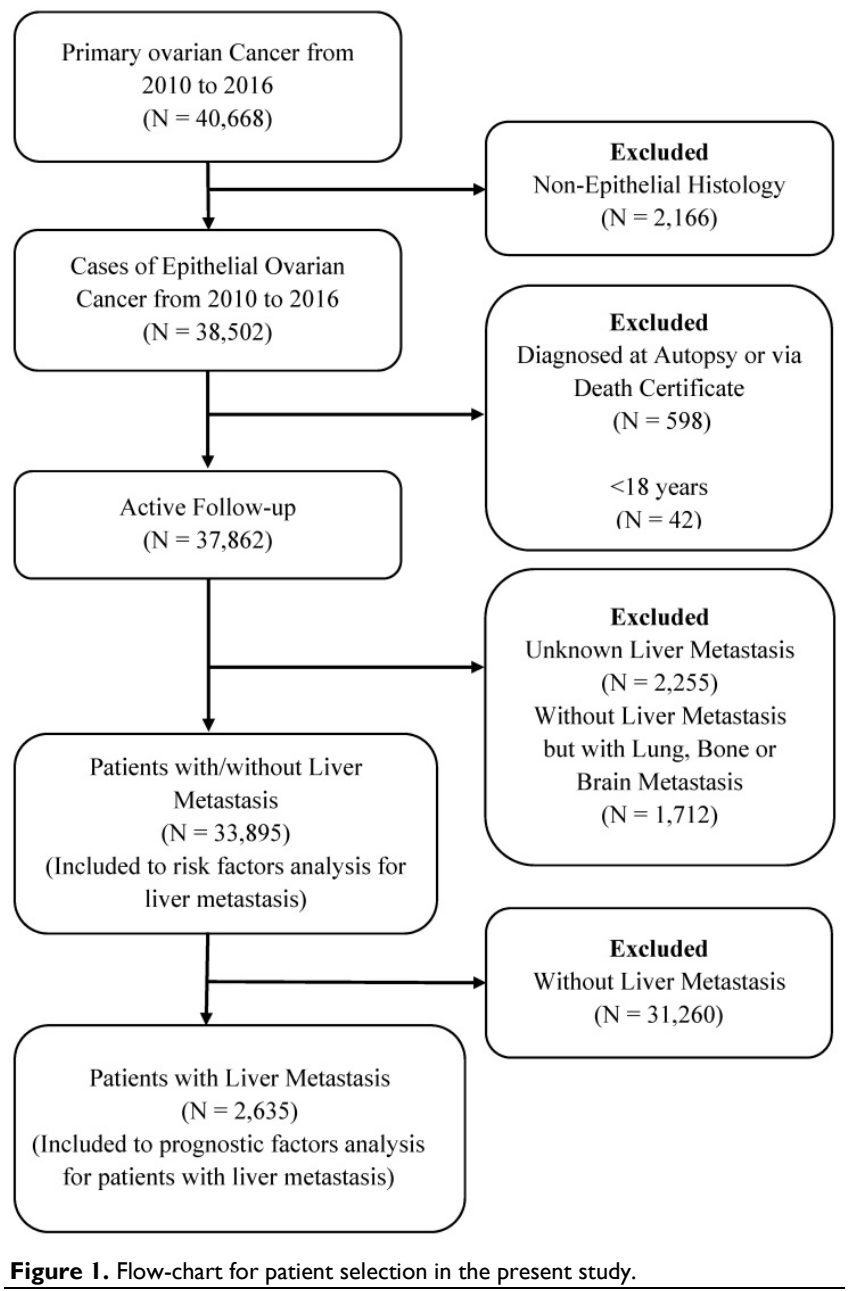

\section{Results}

\section{Demographic and Clinical Characteristics}

Based on selection criteria, a total of 33895 women diagnosed with EOC were involved in the retrospective study cohort (Figure 1). The average age of the women in the entire group was $62.57 \pm 14.21$ years. Most patients were aged 40 and above years $(94.24 \%)$, and $81.97 \%$ were white. Among these patients, 2,635 patients (7.77\%) with EOC and LM had mean survival $(23.73 \pm 24.27$ months), and the mean age was $66.03 \pm 13.64$ years. Detailed characteristics of the subjects are shown in Table 1.

\section{The Incidence of Liver Metastases (LM)}

A total of 2635 primary EOC patients with distant liver Metastases (LM) were diagnosed between the years 2010 and 2016. Univariate analysis of subgroups showed patients $\geq 65$ years of age $(9.65 \%)$ presented with a significantly higher incidence of liver Metastases when compared with patients in lower age groups $\left(x^{2}=145.55, p<0.001\right)$. Furthermore, black $(12.17 \%)$ patients compared with white and others $\left(\chi^{2}=87.96, p<0.001\right)$, unmarried status $(8.78 \%) \quad\left(X^{2}=42.83, \quad \mathrm{p}<0.001\right)$, paired side laterality $(16.87 \%) \quad\left(X^{2}=734.36, \quad p<0.001\right)$, Brenner tumor histology $(17.63 \%)\left(\chi^{2}=939.44, p<0.001\right)$, higher T-stage $(9.65 \%) \quad\left(\chi^{2}=1405.20, \quad p<0.001\right), \quad N$ stage $(12.14 \%) \quad\left(X^{2}=428.72, p<0.001\right)$, poorly differentiated grade III $(6.15 \%)\left(\chi^{2}=819.01, p<0.001\right)$, elevated cancer antigen-125 $(8.61 \%) \quad\left(\chi^{2}=150.60, p<0.001\right)$, surgery status "not performed" $(19.91 \%)\left(\chi^{2}=1792, p<0.001\right)$, patients without chemotherapy $(8.76 \%) \quad\left(\chi^{2}=19.40\right.$, $\mathrm{p}<0.001)$ and patients who received radiotherapy $(12.56 \%) \quad\left(\chi^{2}=12.47, p<0.001\right) \quad$ showed higher liver Metastases incidence than others. However, the incidence of liver Metastases showed no significant difference in insurance status $\left(\chi^{2}=3.94, \mathrm{p}=0.139\right)$; (Table 1).

\section{Associated Risk Factors for Developing LM}

Univariable logistic regression (Table 2) indicated that older age patients $\geq 40$ years, black ethnicity, unmarried status, paired and bilateral tumor location, Brenner tumor histology, higher $\mathrm{T}$ stages T2 and T3, N1 stage, higher grades II, III and IV, elevated CA-125, and receipt of radiotherapy were all positively associated with LM risk.

On multivariable analysis Brenner tumor histology (OR 1.94, 95\%CI 1.39-2.71, $\mathrm{P}<0.001$ ), higher $\mathrm{T}$ stages T2/T1 (OR 2.28, 95\%CI 1.49-3.50, $P<0.001$ ) and T3/T1 (OR 5.65, 95\%CI 3.87-8.24, $P<0.001$ ), $\mathrm{N}$-Stage (OR 1.67, 95\%CI 1.43-1.95, $P<0.001$ ), poorly differentiated grade III/I (OR 2.16, 95\%CI 1.29-3.59, $\mathrm{p}<0.001)$, and undifferentiated; anaplastic grade IV/I (OR 2.04, 95\%CI 1.22-3.43, $P<0.001$ ), and elevated cancer antigen-125 (OR 1.79, 95\% CI 1.19-2.69, $P<0.001$ ) remained independent characteristics associated with de novo LM. 
Table 1. Demographic and clinical characteristics for epithelial ovarian cancer patients diagnosed with and without liver metastases (LM)

\begin{tabular}{|c|c|c|c|c|c|c|c|c|c|}
\hline \multirow{2}{*}{\multicolumn{5}{|c|}{ metastases (LI }} & \multirow{3}{*}{$\begin{array}{l}\text { Subject } \\
\text { characteristics }\end{array}$} & \multirow{3}{*}{$\begin{array}{c}\text { Univariable } \\
\text { OR }(95 \% \mathrm{CI})\end{array}$} & \multirow{2}{*}{\multicolumn{3}{|c|}{ Multivariable }} \\
\hline & & & & & & & & & \\
\hline \multirow{3}{*}{$\begin{array}{l}\text { Subject } \\
\text { characteristic }\end{array}$} & \multicolumn{2}{|c|}{ No. of epithelial ovarian cancer patients } & \multirow[t]{3}{*}{$\chi^{2}$} & \multirow{3}{*}{$\begin{array}{l}P \\
\text { value }^{\text {a }}\end{array}$} & & & $P$-value & OR $(95 \% \mathrm{CI})$ & $P$-value \\
\hline & With LM & Without LM & & & Age, in years & & & & \\
\hline & $(\mathrm{N}=2635,7.77 \%)$ & $(\mathrm{N}=31260,92.23 \%)$ & & & $18-40$ & Ref & 1.00 & Ref & 1.00 \\
\hline Age, in years & $66.03 \pm 13.64$ & $62.28 \pm 14.22$ & 145.55 & $<0.001$ & $41-64$ & $1.48(1.18-1.85)$ & $<0.001$ & $0.80(0.55-1.16)$ & 0.234 \\
\hline $18-40$ & $87(4.45)$ & 1867(95.55) & & & $\geq 65$ & $2.29(1.84-2.86)$ & $<0.001$ & $0.83(0.57-1.21)$ & 0.335 \\
\hline $41-64$ & $1079(6.45)$ & $15641(93.55)$ & & & Race & & & & \\
\hline$\geq 65$ & $1469(9.65)$ & 13752(90.35) & & & White & Ref & 1.00 & Ref & 1.00 \\
\hline Race & & & 87.96 & $<0.001$ & Black & $1.71(1.52-1.93)$ & $<0.001$ & $1.16(0.88-1.53)$ & 0.27 \\
\hline White & 2080(7.49) & $25703(92.51)$ & & & Others & $0.87(0.75-1.00)$ & 0.111 & $1.04(0.80-1.35)$ & 0.75 \\
\hline Black & $341(12.17)$ & $2461(87.83)$ & & & Unknown & NA & NA & NA & NA \\
\hline Others & $208(6.58)$ & 2952(93.42) & & & Marital status & & & & \\
\hline Unknown & $6(4.00)$ & $144(96.00)$ & & & Married & Ref & 1.00 & Ref & 1.00 \\
\hline Marital status & & & 42.83 & $<0.001$ & Unmarried & $1.31(1.21-1.42)$ & $<0.001$ & $1.14(0.98-1.33)$ & 0.090 \\
\hline Married & $1124(6.83)$ & 15330(93.17) & & & Unknown & NA & NA & NA & NA \\
\hline Unmarried & 1395(8.78) & $14500(91.22)$ & & & Insurance & & & & \\
\hline Unknown & $116(7.50)$ & $1430(92.50)$ & & & Insured & Ref & 1.00 & Ref & 1.00 \\
\hline Insurance & & & 3.94 & 0.139 & Uninsured & $1.22(0.99-1.49)$ & 0.059 & $0.90(0.57-1.43)$ & 0.658 \\
\hline Insured & $2481(7.71)$ & 29699(92.29) & & & Unknown & NA & NA & NA & NA \\
\hline Uninsured & $107(9.22)$ & 1054(90.78) & & & Tumor Location & & & & \\
\hline Unknown & $47(8.48)$ & $507(91.52)$ & & & One side & Ref & 1.00 & Ref & 1.00 \\
\hline Tumor Location & & & 734.36 & $<0.001$ & Paired side & $3.70(3.35-4.09)$ & $<0.001$ & $1.29(0.92-1.81)$ & 0.139 \\
\hline One side & $931(5.19)$ & $16998(94.81)$ & & & Bilateral & $1.57(1.43-1.73)$ & $<0.001$ & $1.04(0.88-1.22)$ & 0.644 \\
\hline Paired side & $830(16.87)$ & $4091(83.13)$ & & & Histology & & & & \\
\hline Bilateral & $874(7.91)$ & $10171(92.09)$ & & & Serous & Ref & 1.00 & Ref & 1.00 \\
\hline Histology & & & 939.44 & $<0.001$ & Endometrioid & $0.24(0.19-0.31)$ & $<0.001$ & $1.03(0.71-1.49)$ & 0.862 \\
\hline Serous & $1200(7.02)$ & $15905(92.98)$ & & & Mucinous & $0.49(0.39-0.63)$ & $<0.001$ & $1.22(0.71-2.12)$ & 0.462 \\
\hline Endometrioid & $65(1.78)$ & $3579(98.22)$ & & & Clear cell & $0.36(0.28-0.48)$ & $<0.001$ & $0.72(0.43-1.19)$ & 0.194 \\
\hline Mucinous & $73(3.63)$ & 1940(96.37) & & & Brenner & $2.83(2.54-3.16)$ & $<0.001$ & $1.94(1.39-2.71$ & $<0.001$ \\
\hline Clear cell & $56(2.67)$ & 2044(97.33) & & & Carcinosarcoma & $1.19(0.96-1.48)$ & 0.106 & $1.06(0.72-1.57)$ & 0.766 \\
\hline Brenner & $581(17.63)$ & $2714(82.37)$ & & & Others & $1.64(1.46-1.86)$ & $<0.001$ & $1.28(0.99-1.66)$ & 0.057 \\
\hline Carcinosarcoma & $97(8.27)$ & 1076(91.73) & & & Unknown & NA & NA & NA & NA \\
\hline Others & $399(11.06)$ & $3210(88.94)$ & & & T stage & & & & \\
\hline Unknown & $164(17.15)$ & $792(82.85)$ & & & $\mathrm{T} 1$ & Ref & 1.00 & Ref & 1.00 \\
\hline T stage & & & 1405.20 & $<0.001$ & $\mathrm{~T} 2$ & $3.38(2.69-4.26)$ & $<0.001$ & $2.28(1.49-3.50)$ & $<0.001$ \\
\hline $\mathrm{T} 1$ & $119(1.34)$ & 8747(98.66) & & & $\mathrm{T} 3$ & $7.85(6.51-9.47)$ & $<0.001$ & $5.65(3.87-8.24)$ & $<0.001$ \\
\hline $\mathrm{T} 2$ & $200(4.40)$ & $4345(95.60)$ & & & Unknown & NA & NA & NA & NA \\
\hline $\mathrm{T} 3$ & $1692(9.65)$ & $15840(90.35)$ & & & N stage & & & & \\
\hline Unknown & $624(21.14)$ & $2328(78.86)$ & & & No & Ref & 1.00 & Ref & 1.00 \\
\hline N stage & & & 967.22 & $<0.001$ & N1 & $2.63(2.39-2.87)$ & $<0.001$ & $1.67(1.43-1.95)$ & $<0.001$ \\
\hline No & $1182(5.00)$ & $22459(95.00)$ & & & Unknown & NA & NA & NA & NA \\
\hline N1 & 852(12.14) & $6168(87.86)$ & & & Grade & & & & \\
\hline Unknown & 601(18.58) & $2633(81.42)$ & & & I & Ref & 1.00 & Ref & 1.00 \\
\hline Grade & & & 819.01 & $<0.001$ & II & $2.51(1.63-3.86)$ & $<0.001$ & $1.42(0.83-2.45)$ & 0.201 \\
\hline I & $27(1.06)$ & 2509(98.94) & & & III & $6.63(4.50-9.78)$ & $<0.001$ & $2.16(1.29-3.59)$ & 0.003 \\
\hline II & $94(2.63)$ & $3485(97.37)$ & & & IV & $5.50(3.72-8.14)$ & $<0.001$ & $2.04(1.22-3.43)$ & 0.006 \\
\hline III & $615(6.66)$ & $8617(93.34)$ & & & Unknown & NA & NA & NA & NA \\
\hline IV & $402(5.59)$ & $6792(94.41)$ & & & CA-125 & & & & \\
\hline Unknown & 1497(13.18) & $9857(86.82)$ & & & Normal & Ref & 1.00 & Ref & 1.00 \\
\hline CA-125b & & & 150.60 & $<0.001$ & Elevated & $4.25(3.29-5.48)$ & $<0.001$ & $1.79(1.19-2.69)$ & $<0.001$ \\
\hline Normal & $62(2.17)$ & 2794(97.83) & & & Unknown & NA & NA & NA & NA \\
\hline Elevated & $2022(8.61)$ & 21455(91.39) & & & Surgery & & & & \\
\hline Unknown & $551(7.29)$ & $7011(92.71)$ & & & None & Ref & 1.00 & Ref & 1.00 \\
\hline Surgery & & & 1792.70 & $<0.001$ & Yes & $0.19(0.18-0.21)$ & $<0.001$ & $0.32(0.24-0.34)$ & $<0.001$ \\
\hline None & 1370(19.91) & $5510(80.09)$ & & & Unknown & NA & NA & NA & NA \\
\hline Yes & $1236(4.62)$ & $25540(95.38)$ & & & Chemotherapy & & & & \\
\hline Unknown & $29(12.13)$ & $210(87.87)$ & & & No/Unknown & Ref & 1.00 & Ref & 1.00 \\
\hline Chemotherapy & & & 19.40 & $<0.001$ & Yes & $0.83(0.76-0.90)$ & $<0.001$ & $1.096(0.87-1.377)$ & 0.430 \\
\hline None/Unknown & $887(8.76)$ & $9238(91.24)$ & & & Radiation & & & & \\
\hline Yes & $1748(7.35)$ & $22022(92.65)$ & & & None/Unknown & Ref & 1.00 & Ref & 1.00 \\
\hline Radiation & & & 12.47 & $<0.001$ & Yes & $1.71(1.28-2.31)$ & $<0.001$ & $1.58(0.89-2.80)$ & 0.120 \\
\hline None/Unknown & $2584(7.72)$ & $30905(92.28)$ & & & Abbreviations: $\mathrm{CA}$ & 5: cancer antigen & 5; NA: Nc & vailable; & \\
\hline Yes & $51(12.56)$ & $355(87.44)$ & & & Factors with Unkn & n data were adjus & d for the lo & istic regression mod & \\
\hline
\end{tabular}

Table 2. Univariable and Multivariable Logistic Regression analysis of EOC patients with Liver Metastases 
Table 3. Univariable and Multivariable Cox Regression for analyzing the prognosis factors for epithelial ovarian cancer with liver metastases

\begin{tabular}{|c|c|c|c|c|c|}
\hline \multirow[t]{2}{*}{ Subject characteristics } & \multirow[t]{2}{*}{ Survival month, Median(IQR) } & \multicolumn{2}{|l|}{ Univariable } & \multicolumn{2}{|l|}{ Multivariable } \\
\hline & & HR $(95 \% \mathrm{CI})$ & $P$-value & $\mathrm{HR}(95 \% \mathrm{CI})$ & $P$-value \\
\hline \multicolumn{6}{|l|}{ Race } \\
\hline White & $14(3.0-42.0)$ & Ref & 1 & Ref & 1 \\
\hline Black & $6(2.0-20.0)$ & 1.47(1.29-1.67) & $<0.001$ & $1.14(0.99-1.32)$ & 0.064 \\
\hline Others & $10(2.0-51.0)$ & $1.02(0.68-1.22)$ & 0.794 & $1.17(0.96-1.41)$ & 0.114 \\
\hline \multicolumn{6}{|l|}{ Marital status } \\
\hline Married & $19(4.0-52.0)$ & Ref & 1 & Ref & 1 \\
\hline Unmarried & $7(2.0-30.0)$ & $1.55(1.41-1.71)$ & $<0.001$ & 1.16(1.04-1.30) & 0.007 \\
\hline \multicolumn{6}{|l|}{ Tumor Location } \\
\hline One side & $11(3.0-42.0)$ & Ref & 1 & Ref & 1 \\
\hline Paired side & $4(1.0-15.0)$ & $1.83(1.64-2.04)$ & $<0.001$ & $0.99(0.88-1.12)$ & 0.902 \\
\hline Bilateral & $28(10.0-57.0)$ & $0.63(0.56-0.71)$ & $<0.001$ & $0.93(0.83-1.07)$ & 0.33 \\
\hline \multicolumn{6}{|l|}{ Histology } \\
\hline Serous & $31(10.0-62.0)$ & Ref & 1 & Ref & 1 \\
\hline Endometrioid & $34(8.0-65.0)$ & $0.92(0.65-1.31)$ & 0.654 & $0.87(0.60-1.24)$ & 0.438 \\
\hline Mucinous & $4(2.0-10.0)$ & $4.17(3.22-5.39)$ & $<0.001$ & $2.38(1.82-3.12)$ & $<0.001$ \\
\hline Clear cell & $12(5.0-22.0)$ & $2.04(1.48-2.80)$ & $<0.001$ & $1.83(1.32-2.55)$ & $<0.001$ \\
\hline Brenner & $5(2.0-14.0)$ & $3.13(2.78-3.53)$ & $<0.001$ & $1.44(1.26-1.66)$ & $<0.001$ \\
\hline Carcinosarcoma & $13(3.0-34.0)$ & $1.87(1.45-2.41)$ & $<0.001$ & 1.44(1.11-1.88) & $<0.001$ \\
\hline Others & $4(1.0-19.0)$ & $2.80(2.45-3.20)$ & $<0.001$ & $1.47(1.27-1.70)$ & $<0.001$ \\
\hline \multicolumn{6}{|l|}{ Surgery (Pri.) } \\
\hline None & $3(1.0-11.0)$ & Ref & 1 & Ref & 1 \\
\hline Yes & $36(15.0-71.0)$ & $0.21(0.19-0.23)$ & $<0.001$ & $0.34(0.29-0.39)$ & $<0.001$ \\
\hline \multicolumn{6}{|l|}{ Chemotherapy } \\
\hline None/Unknown & $2(1.0-4.0)$ & Ref & 1 & Ref & 1 \\
\hline Yes & $24(9.0-54.0)$ & $0.20(0.18-0.22)$ & $<0.001$ & $0.30(0.27-0.34)$ & $<0.001$ \\
\hline \multicolumn{6}{|l|}{ Radiation } \\
\hline None/Unknown & $12(2.0-40.0)$ & Ref & 1 & Ref & 1 \\
\hline Yes & $7(3.0-11.0)$ & $1.74(1.30-2.33)$ & $<0.001$ & $1.52(1.12-2.04)$ & $<0.001$ \\
\hline
\end{tabular}

Abbreviations: IQR: interquartile range; Surgery (Pri.): surgery on primary site;

The above factors with Unknown data were removed from Kaplan-Meier and Cox regression models.

\section{Cox Proportional Hazards Regression Analysis}

Variables race, marital status, tumor location, histology types, surgery, chemotherapy, and radiotherapy were considered as potential prognostic factors included in the univariate cox regression model for pre-assessment. Age, tumor grade, cancer antigen-125, T-stage, and N-stage were excluded secondary to a lack of independent association with clinical outcomes. The results of the final multivariable cox model are listed in Table 3.

EOC patients with LM had a median survival of 12 months; $95 \% \mathrm{CI} 11-14, P<0.001$, as compared with the patients without LM (median survival 51.0 months, 95\%CI 50-52, $P<0.001)$. Once patients developed LM, the patient's survival rates plumped. The overall survival rates of EOC patients for 1-year and 3 -year survival were $78 \%$ and $62 \%$, respectively, while survival rates decreased to $49 \%$ and $27 \%$ respectively after LM diagnosis ( $P<0.001$, Figure $2 \mathrm{~A})$. Univariate cox regression analysis showed, unmarried $(P<0.001$, Figure $2 B)$, black race $(P<0.001$, Figure 2C), paired site tumor location $(P<0.001$, Figure $2 \mathrm{D})$, non-serous histology types $(P<0.001$, Figure $2 \mathrm{E})$, and receipt of radiotherapy $(P<0.001$, Figure $2 \mathrm{H})$ was adversely associated with overall survival.
Conversely, chemotherapy $(P<0.001$, Figure $2 \mathrm{~F})$, and surgical treatment of the primary site $(P<0.001$, Figure $2 \mathrm{G})$ were positively associated with improved overall survival $(P<0.001)$. The final Cox Model included independent prognostic factors with adjusted subgroups showed, when patients with married status were defined as the referent, the Hazards Ratio (HR) for disease progression in unmarried women was 1.16 (95\%CI 1.04-1.30, $P<0.001)$, with median survival and interquartile range (median 7.0; IQR 2.0-30.0) months. Those with non-serous histology types, the mucinous (HR: 2.38; 95\% CI 1.82-3.12, $P<0.001$ ), clear cell (HR: 1.83; 95\%CI: 1.32-2.55, $P<0.001)$, Malignant Brenner Carcinoma, NOS, (HR: 1.44; 95\% CI 1.26-1.66, $P<0.001)$ and Carcinosarcoma (HR: 1.44; 95\% CI 1.11-1.88, $P<0.001$ ) had a higher risk of disease prognosis. Receipt of radiotherapy was positively related to increased risk of death (HR: 1.52; 95\% CI 1.12-2.06, P<0.001). Besides, patients who received Neoadjuvant chemotherapy (HR: 0.30; $95 \% \mathrm{CI} 0.27-0.34, P<0.001)$ and with surgical treatment of the primary site (HR: 0.34; 95\%CI 0.29-039, $P<0.001)$, presented longer overall survival than those who did not receive treatment of chemotherapy. The median survival of the patients who received 
chemotherapy (Median survival: 24.0; IQR 9.0-54.0 months) and with surgery (Median survival: 36.0 IQR 15.0-71.0 months) as compared to the patients who did not receive chemotherapy (Median survival: 2.0; IQR 1.0-4.0 months) and surgery (Median survival: 3.0; IQR 1.0-11.0 months) respectively.

\section{Discussion}

To our knowledge, this is the first large population-based study to explore the demographic and clinical characteristics, potential risks, and prognostic factors of LM from EOC that have been analyzed premortem up to this point. Based on our

A
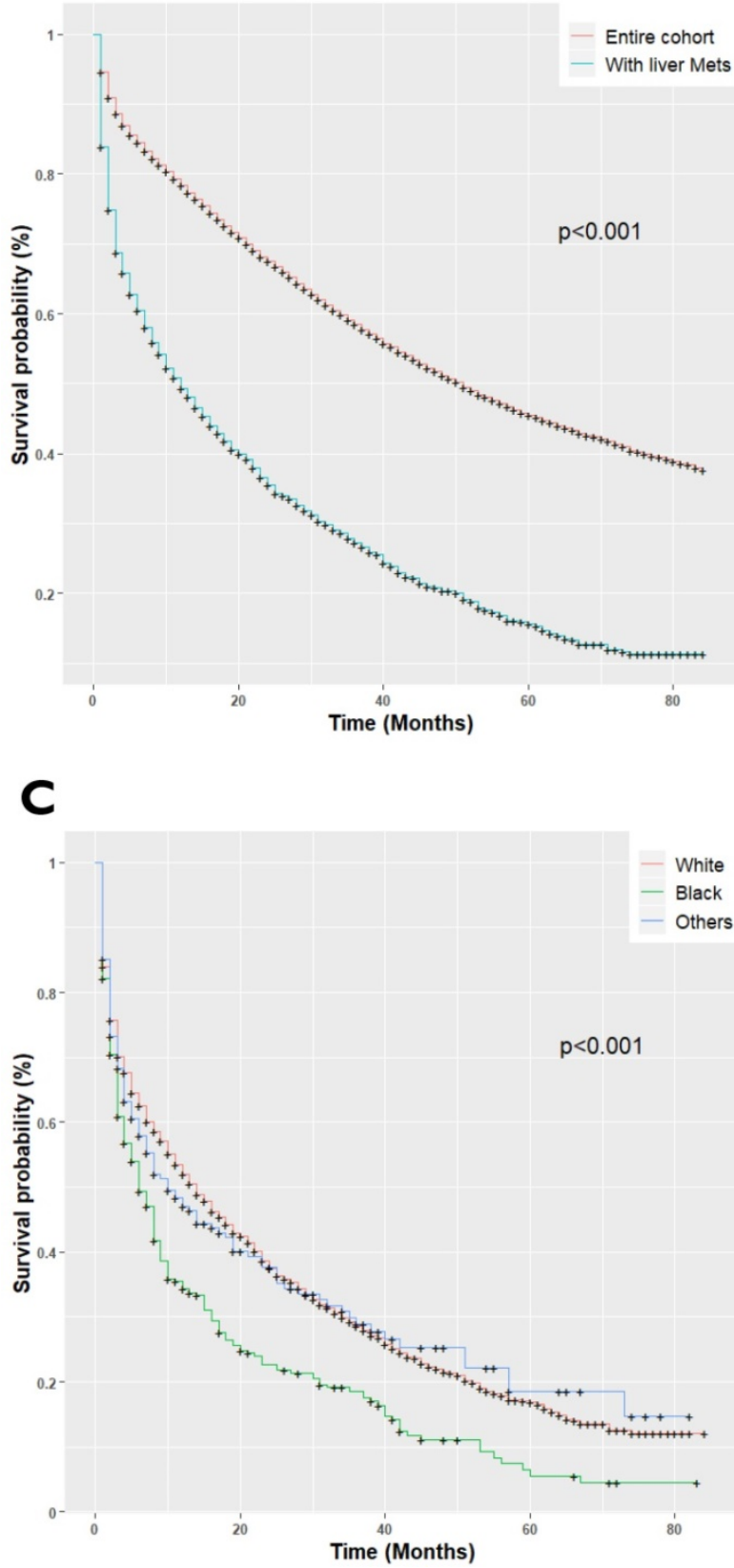

retrospective cohort, $7.77 \%$ of primary EOC patients initially diagnosed with de novo LM. Winter et al. reported that Hepatic parenchymal Metastases accounted for $18 \%$ and was the second most common cause of stage-IV disease in a large GOG study [19]. The metastatic rate to the liver in our study was lower than the previous studies, as most of the studies restricted to the distant stage (stage-IV) disease. The prognostic role of the LM pattern in EOC patients remains to be explained. Potential risk factors identification is needed to investigate for LM progression and screening performance among high-risk EOC patients.

B
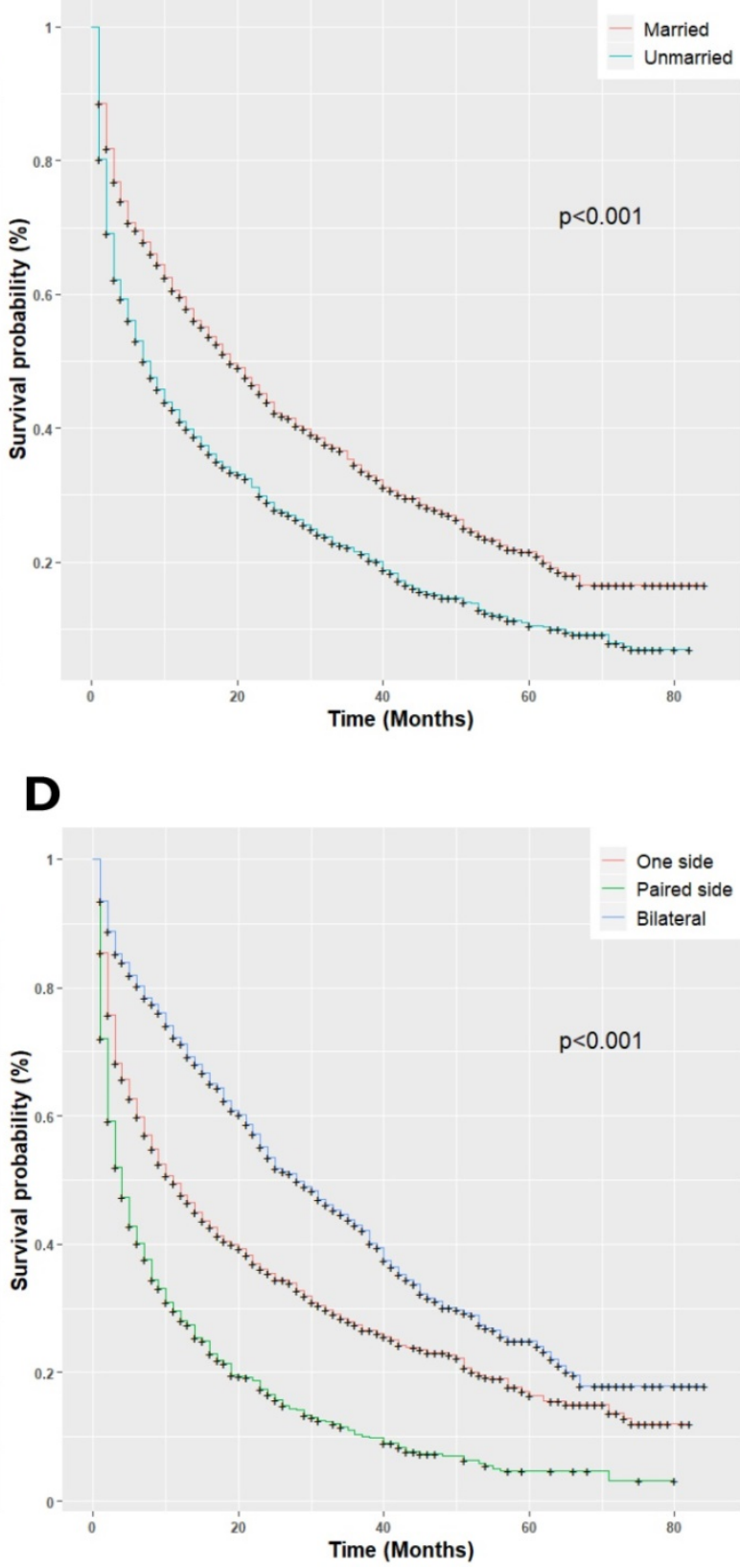

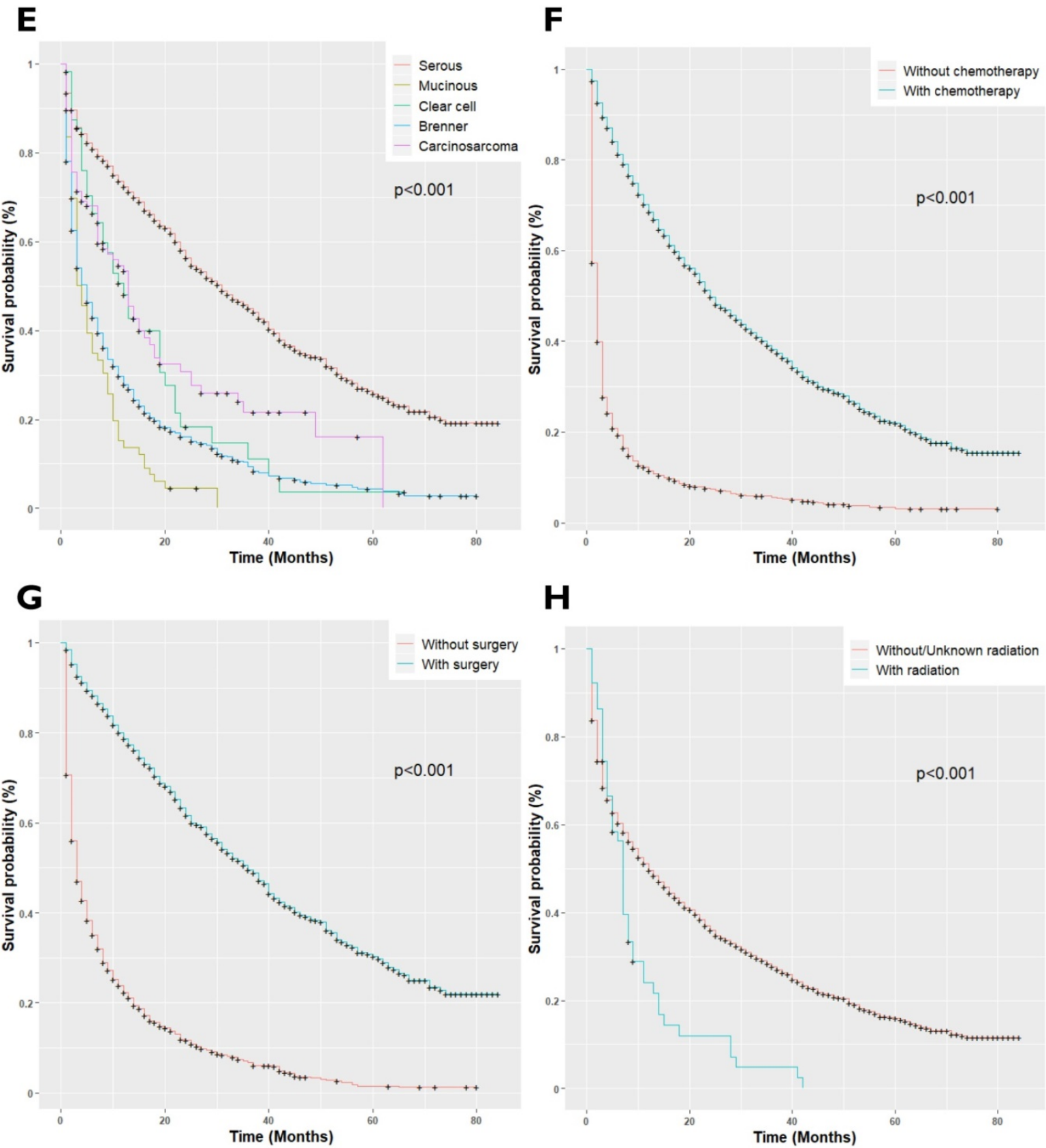

Figure 2. (A) Kaplan-Meier survival curves for patients with and without Liver metastases; (B) Kaplan-Meir survival curves for patients with Marital Status; (C) Kaplan-Meier survival curves for patients with race/ethnicity; (D) Kaplan-Meier survival curves for patients with tumor location; (E) Kaplan-Meier survival curves for patients with histology types; (F) Kaplan-Meier survival curves for patients with Neoadjuvant chemotherapy status; (G) Kaplan-Meier survival curves for patients with surgery status; (H) Kaplan-Meier survival curves for patients with radiotherapy status.

The current study identified older age, black race, unmarried status, paired site tumor location, Brenner tumor histology type, higher T-stage, $\mathrm{N}$ stage, poorly differentiated grades III and IV, cancer antigen-125, and receipt of radiotherapy was significantly associated with de novo LM development. Our findings corroborate the findings of previous studies [20-23]. Mizuno et al. found by analyzing 223 EOC patients that non-serous tumor histology types, the clear cell and mucinous were significantly associated with worse prognosis and a higher rate of early deaths compared to the Serous and endometrioid histology tumors $(P<0.001)$ [24]. The results of the current study provide further support to the evidence suggesting non-serous histology types have reduced survival, with the median overall survival of 4-13 months as compared to 31-34 months in serous and endometrioid histology types [25].

In our study, EOCLM patients who underwent 
primary surgery had higher survival compared to those who did not experience this procedure. Almost $47 \%$ of the patients underwent surgical cytoreduction of the primary site; the data still suggest that the surgical approach of primary cytoreduction in advanced-stage disease is interrelated with improved overall survival of EOCLM patients. Winter et al. observed a prolonged survival in EOCLM patients who received primary cytoreductive surgery [19]. Additionally, prior studies also suggested that cytoreductive surgery of primary site of EOC patients with FIGO stage-IV disease more likely to have immense advantages $[10,26,27]$.

Chemotherapy is commonly employed in patients with advanced-stage EOC patients. $66.33 \%$ of patients in our study received chemotherapy. Our results suggest that chemotherapy offers enormous benefits in the adjuvant treatment of women with EOCLM. In the current analysis, it was observed that chemotherapy admiration was associated with highly improved survival. It is prominent since this strengthens the reason for better assumptions for median progression-free and overall survival. The study findings are similar to those in other regional or multicenter studies that analyzed distant-stage epithelial ovarian carcinomas separately [28-33].

One of the main strengths of the current study is the fact that it presents a large number of LM patients from nationwide registries. Moreover, the study period spanned seven years with active follow-up. Conversely, several limitations should be noted. First of all, the SEER database does not provide information on the administration of hepatic resection during cytoreductive surgery for primary EOCLM patients. Comorbidity profiles of the patients, such as hepatic involvement, the liver parenchymal invasion, hematogenous dissemination, and patient's preference to receive primary or secondary metastatic surgery, which may partially affect the precision of the results. Secondly, our results showed a strong association between the surgery of the primary site and improvement in survival among EOCLM patients. However, the information regarding the history of the disease, and treatment morbidity is lacking. Thirdly, the SEER database does not provide information regarding those EOC patients who have developed liver metastases later during follow-up. Lastly, one of the limitations of the current research was the lack of validation of the present study through an independent cohort as actively followed EOC patients with LM lacked in other databases. Therefore, the observed LM of the current research has to be interpreted as an underestimate of the real figure.

In conclusion, our study clarified the epidemiology of EOCLM in US women. The survival of women with liver Metastases who underwent surgery of primary site and chemotherapy exceeds that of patients without surgery or chemotherapy. Besides, radiation therapy was negatively associated with the overall survival of the patients. The prognosis of EOCLM patients is poor, with a median survival of 12 months. Non-serous histology-types, the mucinous, clear cell, Malignant Brenner Carcinoma, NOS, and Carcinosarcoma, were positively associated with overall death. Comparatively, good survival was observed in the white race, married women, and those who had one-side or bilateral tumors. The limitations of the present study support the need for related studies to further confirm the results in the future.

\section{Acknowledgments}

We want to thank the SEER public database for making the ovarian cancer data available. The present study was sponsored by the National Natural Science Foundation of China under grant number: 81773551.

\section{Ethics approval and consent to participate}

The SEER is a publically available database. The data does not require informed patients' consent. Our study used de-identified secondary data, which was deemed exempt from review by the ethics board of the Harbin Medical University.

\section{Competing Interests}

The authors have declared that no competing interest exists.

\section{References}

1. Jemal A, Siegel R, Ward E, Murray T, Xu J, Thun MJ. Cancer Statistics, 2007. CA Cancer J Clin. 2007; 57: 43-66.

2. Jelovac D, Armstrong DK. Recent Progress in the Diagnosis and Treatment of Ovarian Cancer. 2011; 61: 183-203.

3. Torre LA, Trabert B, Desantis CE, et al. Ovarian Cancer Statistics , 2018. 2018; 284-96.

4. Ataseven B, Chiva LM, Harter P, Gonzalez-Martin A, du Bois A. FIGO stage IV epithelial ovarian, fallopian tube and peritoneal cancer revisited. Gynecol Oncol. 2016; 142: 597-607.

5. Cancer O. Metastatic Patterns in Histologic Variants. Cancer. 1989; 1508-13.

6. Gasparri ML, Grandi G, Bolla D, et al. Hepatic resection during cytoreductive surgery for primary or recurrent epithelial ovarian cancer. J Cancer Res Clin Oncol. 2016; 142: 1509-20.

7. Lu M, Li Z, Yang C, et al. Sites of distant metastases and overall survival in ovarian cancer: A study of 1481 patients. Gynecol Oncol. 2018; 150: 460-5.

8. Bacalbasa N, Dima S, Brasoveanu V, et al. Liver resection for ovarian cancer liver metastases as part of cytoreductive surgery is safe and may bring survival benefit. World J Surg Oncol. 2015; 13: 4-9. Available at:

9. Invasion LP, Ishill N, Soslow RA, Chi DS. Perihepatic Metastases from Ovarian Cancer: Sensitivity and Specificity of CT for the Detection of Metastases with and Those without Purpose : Methods : Results : Conclusion : 2008; 248: 511-7.

10. Bristow RE, Puri I, Chi DS. Cytoreductive surgery for recurrent ovarian cancer: A meta-analysis. Gynecol Oncol. 2009; 112: 265-74.

11. Poveda Velasco A, Casado Herráez A, Cervantes Ruipérez A, et al. Treatment guidelines in ovarian cancer. Clin Transl Oncol. 2007; 9: 308-16.

12. Hussain SM, Semelka RC. Hepatic imaging: Comparison of modalities. Radiol Clin North Am. 2005; 43: 929-47.

13. Blair AR, Casas CM. Gynecologic Cancers. Prim Care - Clin Off Pract. 2009; 36: 115-30. 
14. Liu PS, Francis IR. Hepatic imaging for metastatic disease. Cancer J. 2010; 16: 93-102.

15. World Health Organization (WHO). Icd-O-3. World Heal Organ. 2000; 240.

16. Cates JMM. The AJCC 8th edition staging system for soft tissue sarcoma of the extremities or trunk: A Cohort study of the SEER database. JNCCN J Natl Compr Cancer Netw. 2018; 16: 144-52.

17. Peres LC, Cushing-Haugen KL, Köbel M, et al. Invasive Epithelial Ovarian Cancer Survival by Histotype and Disease Stage. J Natl Cancer Inst. 2019; 111: 60-8.

18. Wickham H. ggplot2: Elegant Graphics for Data Analysis. Journeal Stat Softw. 2017; 80: 1-4.

19. Winter WE, Maxwell GL, Tian C, et al. Tumor residual after surgical cytoreduction in prediction of clinical outcome in stage IV epithelial ovarian cancer: A Gynecologic Oncology Group study. J Clin Oncol. 2008; 26: 83-9.

20. Cormio G, Rossi C, Cazzolla A, et al. Distant metastases in ovarian carcinoma. Int J Gynecol Cancer. 2003; 13: 125-9.

21. Loizzi V, Rossi C, Cormio G, Cazzolla A, Altomare D, Selvaggi L. Clinical features of hepatic metastasis in patients with ovarian cancer. Int J Gynecol Cancer. 2005; 15: 26-31.

22. Karagol H, Saip P, Eralp Y, et al. Factors related to recurrence after pathological complete response to postoperative chemotherapy in patients with epithelial ovarian cancer. Tumori. 2009; 95: 207-11.

23. Wang F, Zhao X, Tan W, Liu W, Jin Y, Liu Q. Early identification of recurrence in ovarian cancer: A comparison between the ovarian cancer metastasis index and CA-125 levels. PeerJ. 2018; 2018.

24. Mizuno M, Kajiyama H, Shibata K, et al. Prognostic value of histological type in stage IV ovarian carcinoma: A retrospective analysis of 223 patients. Br J Cancer. 2015; 112: 1376-83.

25. Peres LC, Cushing-haugen KL, Ko M, et al. Invasive Epithelial Ovarian Cancer Survival by Histotype and Disease Stage. 2019; 111: 1-9.

26. Wimberger $\mathrm{P}$, Wehling $\mathrm{M}$, Lehmann $\mathrm{N}$, et al. Influence of residual tumor on outcome in ovarian cancer patients with FIGO stage IV disease. Ann Surg Oncol. 2010; 17: 1642-8.

27. Rauh-Hain JA, Melamed A, Wright A, et al. Overall survival following neoadjuvant chemotherapy vs primary cytoreductive surgery in women with epithelial ovarian cancer: Analysis of the National Cancer Database. JAMA Oncol. 2017; 3: 76-82.

28. Barber EL, Dusetzina SB, Stitzenberg KB, et al. Variation in neoadjuvant chemotherapy utilization for epithelial ovarian cancer at high volume hospitals in the United States and associated survival. Gynecol Oncol. 2017; 145: 500-7.

29. Meyer LA, He W, Sun CC, et al. Neoadjuvant chemotherapy in elderly women with ovarian cancer: Rates of use and effectiveness. Gynecol Oncol. 2018; 150: 451-9.

30. Lee YJ, Chung YS, Lee JY, et al. Impact of increased utilization of neoadjuvant chemotherapy on survival in patients with advanced ovarian cancer: Experience from a comprehensive cancer center. J Gynecol Oncol. 2018; 29: 110.

31. Aletti GD, Dowdy SC, Podratz KC, Cliby WA. Analysis of factors impacting operability in stage IV ovarian cancer: Rationale use of a triage system. Gynecol Oncol. 2007; 105: 84-9.

32. Vergote I, Coens $C$, Nankivell M, et al. Neoadjuvant chemotherapy versus debulking surgery in advanced tubo-ovarian cancers: pooled analysis of individual patient data from the EORTC 55971 and CHORUS trials. Lancet Oncol. 2018; 19: 1680-7.

33. Vergote I, Tropé CG, Amant F, et al. Neoadjuvant Chemotherapy or Primary Surgery in Stage IIIC or IV Ovarian Cancer. N Engl J Med. 2010; 363: 943-53.

34. Surveillance, Epidemiology, and End Results (SEER) Program (www.seer.cancer.gov) SEER ${ }^{\star}$ Stat Database: Incidence - SEER 18 Regs Custom Data (with additional treatment fields), Nov 2018 Sub (1975-2016 varying), released April 2019, based on the November 2018 submission. 